\title{
Wireless Power Transmission
}

\author{
${ }^{1}$ Sudha Bhutada(Senior Lect.), ${ }^{2}$ Punit Maheshwari(R\&D Eng.) \\ ${ }^{I}$ EE Dept.SV Polytechnic College Bhopal, India \\ ${ }^{2}$ VLSI Dept. Navigator Technologies Bhopal, India
}

\begin{abstract}
The concept of wireless power transmission method is as old as we are using electricity. N. Tesla the father of wireless power transmission was the first to give an idea of wireless power transmission and he was keenly interested in lightning the world without using wires (WPT).Since then series of experiments were made to make feasible transmission of power where it is not possible and commercially viable by advances, inventions in electronic devices in this field. In this paper we present the concept of wireless power transmission from one place in turn to another. This concept is mainly used for reducing the transmission and distribution losses. This in turn reduces the cost of transmission and distribution and also the cost of electrical energy for the consumers. In this paper we discussed the methods, recent developments in WPT, its merits, demerits and applications.
\end{abstract}

Keywords: Wireless Power Transmission WPT, Radio Frequency RF

\section{Introduction}

We know our present system of overhead transmission and distribution has biggest disadvantage is percentage of power loss is around 26 to $28 \%$ during transmission and distribution which is because of resistance of wires of grid. WRI (World resources Institute) says that India's electricity grid has highest T\&D losses in the world around 27\%. By Indian government agencies it is around 30 to $40 \%$ or even greater due to technical losses and power theft. More over power demand is increasing day by day. There is a need of electricity in every nook and corner of the world. So we need an alternative solution to the problem which provides much higher efficiency, low transmission cost, avoid power theft, eco friendly power, efficient and reliable, reduces the dependence on wires and batteries. For this only solution is wireless power transmission system.

Wireless power or wireless transmission of electrical energy from a power source to an electrical load without man made conductors. It is useful in cases where interconnecting wires are inconvenient, hazardous or impossible. It is carried out using direct induction followed by resonant magnetic induction, electromagnetic radiation in the form of microwaves or lasers and electric conduction through media.

This system now a day is very popular all over the world. Radio waves are the energy and people use them to send and receive cell phone, TV, radio, Wi-Fi signals day to day. This technology now a day has a wide foothold all over the world. This technology today has matured enough to allow us a new means to power our mobile and gadgets.

\section{A. History}

\section{Overview}

Wireless power transmission beginning started from-

1826- Andre-Marie Ampere develops Ampere's circuital law showing that electric current produces a magnetic field.

1831- Michael Faraday develops Faraday's law of electromagnetic induction describing the electromagnetic force induced in a conductor by a time varying magnetic flux.

1836- Nicholas Callan invents the electric transformation also known as induction coil.

1891- Nikola Tesla demonstrates wireless energy transmission

by means of electromagnetic induction using high tension coil before the American Institute of Electrical Engineers at Colombia College.

1893- Tesla demonstrates wireless illumination of phosphorescent lamps at the Columbian exposition in Chicago.

1893- Tesla proposes the wireless transmission signals before a meeting of National Electric Light Association in St. Louis (possibility of WPT)

1897- Tesla got the first patent registered for wireless transmission.

1964- William Brown demonstrated a model helicopter flown with a beam of microwave sent from the ground.

2008- Intel reproduces Tesla's 1894 electro dynamic induction and wirelessly powered a bulb with 75\% energy efficiency.

2010- Haier group world's first completely LCD television based on Tesla's electro dynamic principle. 
Since 1800's series of researchers performed experiments to make WPT technology into reality.

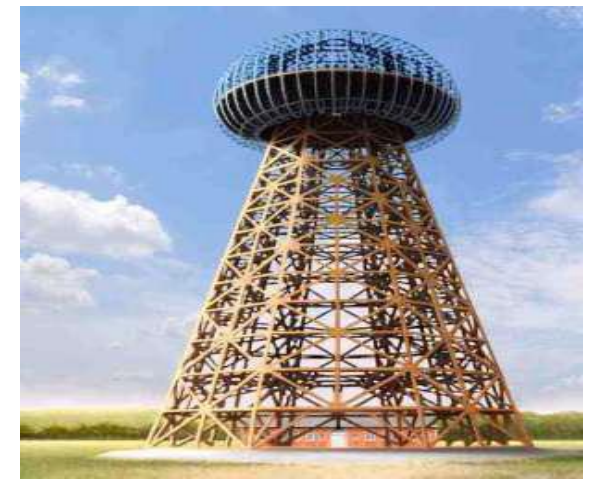

A transmitter broadcasts a low power radio frequency

Figure1.The 187-foot Wardenclyffe Tower

\section{B. Definition}

Wireless power or Wireless energy transmission is transmission of electrical energy from a power source to electrical load without man-made conductors. A wireless data transmission eliminates the need of wires. With the increase in wireless data application the need of wireless energy transfer technology has increased. It is the latest technology for direct powering and wireless charging of cell phones, game controllers, laptop computers, mobile robots, electric vehicles, flat screen TV's ,digital picture frames hanging on the wall without requiring adaptor. Industrial systems, medical devices made more reliable by eliminating wiring and replaceable batteries.

\section{Classification of WPT}

WPT has been classified mainly into five categories as-

1 Magnetic resonance

2 Inductive coupling

3 Radio frequency

4 Microwave

5 Laser

\section{Magnetic resonance-}

It uses near field inductive coupling through magnetic field. This technology is not much developed yet and needs further research.

\section{Inductive coupling-}

Most developed form of WPT, operates in lowest region of electromagnetic spectrum. It is based on electromagnetic induction principle. It is a term for interdependence of time varying electric and magnetic fields. The oscillating magnetic field produces a magnetic and electric field. An electric current flow through a wire creates a circular magnetic field around the wire. Bending the wire into a coil amplifies the magnetic field, the more the loops the coil makes, the bigger the field will be. If a second coil of wire is placed in the magnetic field that has been created, the field can induce a current in the wire.

This approach has already seen in multiple commercial applications. E.g. - recharging pads, designed to recharge several devices at once (like the splash power recharging mat and Edison's Electric power desk). This method works only on small distances. However distances can be increased by resonant coupling. Electrostatic induction or capacitive coupling uses capacitive coupling from opposing plane electrodes. Its first commercial application as ,"AIR VOLTAGE for ipad2" by Murata manufacturing company in 2011.

\section{Radio Frequency-}

A transmitter broadcasts a low power radio frequency signals at a specific frequency across a several feet of empty space. A receiver built into one or more remote devices captures enough energy to continuously recharge batteries or to power devices directly. The basis of radio wave transmission technology is converting RF signals to DC electrical current powered from either an international or ambient power sources.

E.g. - Wi-Fi, mobile networks, laptops, remote sensors. This method provides a continuous power source up-to a distance of 30 meters. 


\section{Microwave-}

This technology is still to be commercialized. This is a far field technology. This technique is mainly dependent on radioactive techniques.

Here wave are either broadcasted in the form of narrow beam transmission of radio or light waves(high power transfer).Tesla gave this concept in 1980's by constructing large Wardenclyffe Tower to mainly transfer the energy for long distances.

MPT (microwave power transmission) involves the conversion of energy into microwaves and then transfer the waves through the rectenna (rectifier and antenna) from the transmitter and received at the receiver which will be converted into the conventional electrical power.

Steps involved are-

- Conversion of electrical energy into microwave energy.

- Receiving microwave energy using rectenna.

- Conversion of microwave energy to electrical energy.

Many longer range power transmission ideas also rely on using rectifying antennas or rectennas. Rectennas represent a very efficient way to convert microwaves to electricity. They are usually made an array of dipole antenna, which have positive and negative poles. These antennae connect to semiconductor diodes. Basically the antenna array collects microwave energy and converts it into direct current electricity.

E.g. - Solar power stations on the moon, Solar power satellite

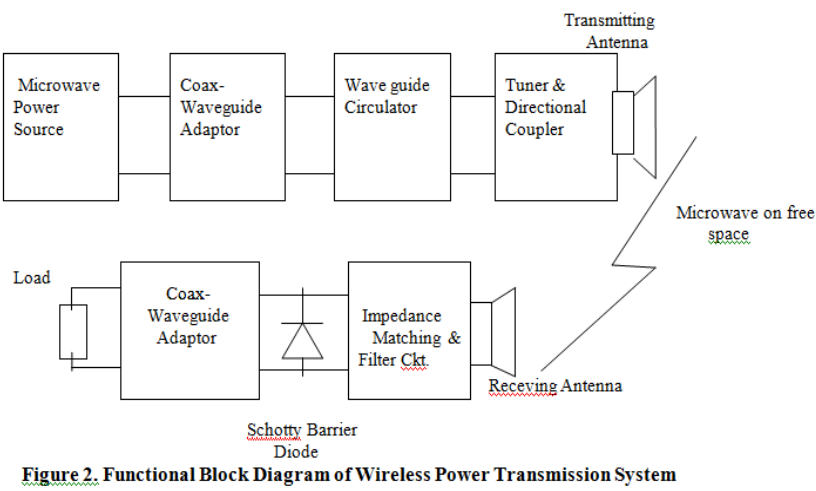

\section{Laser technology-}

This is an efficient way of power transmission. It uses the same possibility as microwave wireless transmission but has energy emission of high frequency and is coherent.

Research organizations like Nassau, Entech, etc or working in this field. Moreover in this technology aperture collection efficiency which is, that antenna can be small sized. It is simple like photovoltaic cell. Therefore this method is more efficient than microwave power transmission. Power transfer can be achieved by converting energy into a laser beam and then pointing it at a solar cell. It transforms over large areas, small size components are used, no interference, access controllable. Main drawback is conversion of electricity to light and back is inefficient, atmospheric absorption causes losses. E.g.- of this technology is supplying an unmanned aircrafts using invisible ground based laser, as demonstrated by the researchers of NASA's Marshall Space Flight Center Hurtsville, Ala. Other applications powering extra-terrestrial reveres and aircrafts providing more power to satellites orbiting earth, power transmissions to locations not otherwise accessible.

\section{Applications}

Wireless power in the field of consumer electronics- automatic wireless charging of mobile electronics like phones, laptops, game controllers while devices are in use. Helps in direct wireless powering of stationary devices like flat screen TV's, digital picture frames, home theatre accessories, wireless loudspeakers etc. eliminating wiring and cables. Direct wireless powering of desktop PC peripherals, wireless mouse, keyboard, printers, speakers etc and eliminating batteries and cables.

Wireless power in the field of Industrial appliances- Direct wireless power for rotating and movable joints like robots, packaging machinery, assembly machinery, machine tools, in harsh environment like drilling, mining, underwater etc for sensors and actuators, mobile robots, automatic guided vehicles, cordless tools and instruments, in medical devices like pacemakers, defibrillator etc eliminating the use of expensive wiring and replacement of batteries. It is also used in automatic charging of smart cards.

Wireless charging in the field of electric vehicles like golf carts, industrial vehicles, electric passengers etc.

Wireless charging has an application in the field of military systems like battery power mobile devices, sensors, mobile robots, aircrafts etc. 


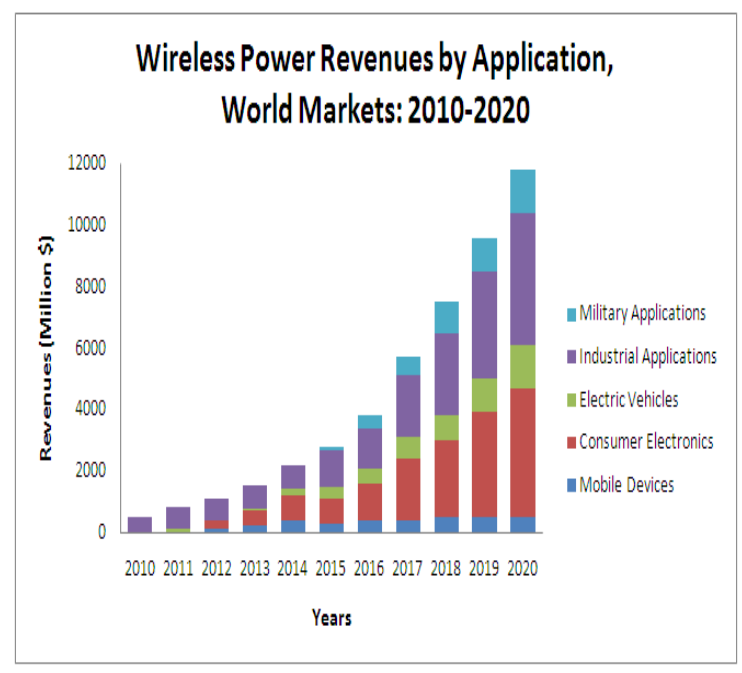

\section{Current Status Of Wireless Power}

Industries are very keen in finding latest wireless power technologies to improve reduce costs, maintain connectivity, versatile, and eliminate the need to replace batteries. Power plant industries are interested in using WPT as a power source for sensors and transducers. In electronics industry wireless power charging and transmission have shown a great promise for enabling plug-free and in many cases contactless charging for wide range of devices from mobile phones to electric vehicles to unmanned aircraft.

Techniques have evolved to transmit dozens of watts over dozens kilometers.

An active and emerging industry like General motors and General electric as well as startups like Witricity, Power cast, Pure Energy solutions and Power mat are coming up.

The maturity of technology depends upon degree of commercialization of a specific technology. WPT can be commercial, near commercial and research. In WPT Commercial and near commercial are in short ranges or very short range. Electromagnetic induction is the commercialized WPT technology today.

According to Pike research worldwide revenues from wireless power transmission and charging systems will reach $\$ 11.8$ billion by 2020 with a compound growth rate (CGAR) of $36 \%$.

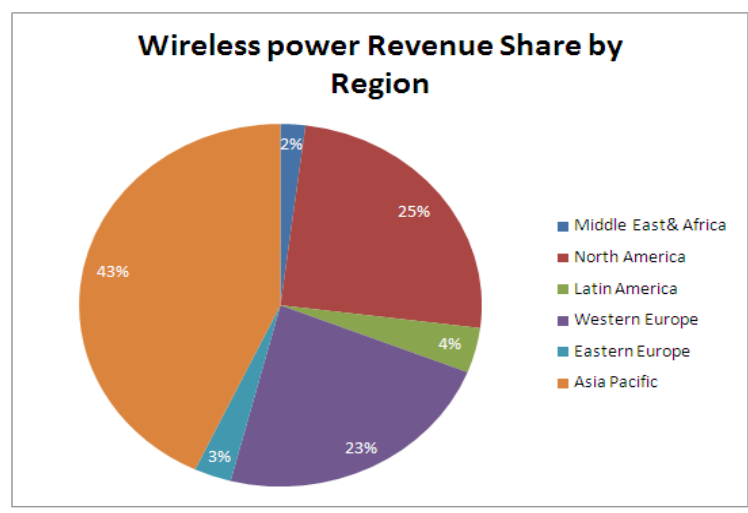

\section{Merits}

This system has evolved to transmit dozens of power over km wirelessly

This system eliminates the high tension line cables, towers and sub stations which are seen as not very efficient way of energy transmission.

This system will globally connect the entire power system.

It offers a range of simple and convenient charging modalities from a mat that will charge several devices in a given range.

The cost of transmitting and receiving the energy becomes less thereby reducing the tariff rate.

Loss of power through transmission is negligible.

The natural hazards like earthquakes, landslides, floods, cannot cut the power in WPT system.

The power failure due to short circuit of cables or faults in lines will not occur.

The space occupied by infrastructures like power house, dam, substations and transmission grid will be eliminated. 
The driver cost i.e. the overall cost per watt or kilowatt (tariff) for charging will go down as physical chargers and adapters are removed.

This technology advances in wireless power for both cost savings and higher consumer satisfactions in electrical vehicle market, industrial applications and military applications.

This technology is clean energy and carbon reductions as reduction in cords, adaptors, and wall outlets.

Plug in system in market has made consumer to adopt electric vehicles.

This system reduces green house gas emissions by eliminating the need for copper wire transmission grids. Transporting power from remote generation sources like PV arrays, wind plant etc. Collecting and utilizing micro power from ambient sources like cellular networks. Replacing costlier and carbon intensive electric sources like diesel generators. Massive solar arrays into geosynchronous orbit and beaming power back to earth in the form of microwaves.

\section{Demerits}

Initial cost is high for its practical implementation. As microwave is used interference is high. In laser technique attenuation losses, radiation losses etc. are the concerned factors. The major concern remains the safety of the waves it emits the biological impacts.

\section{Conclusion}

The concept of WPT, technological development, advantages, disadvantages, applications of WPT are also discussed. This concept of power transmission wirelessly is a great boon to mankind. With the adoption of wireless power charging and transmission devices particularly for mobile devices, consumer electronics, and electric vehicles, will climb steadily over the next five years and then accelerate rapidly as prices fall and this system is used in many everyday products. We can expect wonder in this field if all the conditions are favorable.

\section{References}

[1]. Matsumoto, H.N. Kaya, I. Kimura, S. Miyatake, M. Nagatomo, and T. Obayashi, MINIX Project toward the Solar Power Satellites --- Rocket Experiment of microwave energy transmission and associated plasma physics in the ionosphere, ISAS space energy symposium,pp 69-76, 1986. (references)

[2]. J.J Schelesak, A. Alden and T. Ohno, A microwave powered high altitude platform, IEEE MTT-S Int. Symp. Digest, pp 283-286, 1998

[3]. L. W. Epp, A. R. Khan, H. K. Smith, and R.P.Smith, “A compact dual- polarized 8.51-GHz rectenna for high-voltage (50 V)actuator applications," IEEE Trans. Microwave Theory Tech., vol. 48,pp. 111-120,2000 .

[4]. J.C. Lin, "Biological aspects of mobile communication fields," Wireless Networks, vol. 3,pp. 439-453, 1997.

[5]. T-WYoo and K. Chang, "Theoretical and experimental development of 10 and 35 GHz rectennas," IEEE Trans. Microwave Theory Tech., vol.40,pp. 1259-1266, 1992.

[6]. P. Koert and J.T. Cha, “35 GHz rectenna development," in Proc. 1 1993, pp. 457-466.

[7]. T.-W. Yoo, "Experimental and theoretical study on $3 \mathrm{GHz}$ RF-to-DC power conversion receiver for millimeter-wave beamed power transmission," Ph.D. dissertation, Dept. of Electrical Engineering, Texas A\&M Univ., Dec. 1993. 